\title{
13 Die Literatur als Ort der Aufbewahrung des indirekten Sprechens aus der Konversation: Honoré de Balzac
}

\subsection{Die veränderte Kommunikationssituation im 19. Jahrhundert}

Die Sprache der Marquise de Merteuil aus den Liaisons dangereuses hat gezeigt, wie man sich in einem aristokratischen Umfeld und einer immer noch von der freien Zeit der aristokratischen Lebensweise geprägten Konversation den modernen Gegebenheiten anpassen kann. Utilitarismus und Beschleunigung des öffentlichen Lebens sind die beiden Faktoren, die die Merteuil aufgreift und zu Maßstäben ihres Sprechens macht. Beide Kriterien führen zu einer Konzentration der Sprecherleistung: Da man in einer Epoche der Beschleunigung sowie der geforderten Effizienz umso schneller reagieren muss, ist eine besonders rational geplante Vorbereitung auf mögliche Sprechsituationen sowie eine Einübung der Prinzipien der Rede zwecks Habitualisierung nötig. Und umso wichtiger ist das notwendige Maß an Verstellung, die das indirekte Sprechen ermöglicht, da dieses dem Gesprächspartner größere Verstehensleistungen auferlegt und zugleich Spielräume der Reaktion ermöglicht sowie Ausweichmöglichkeiten eröffnet. Insbesondere die in der Regel spontan und unwillkürlich erfolgende Körpersprache muss, wie der Fall der Merteuil zeigt, strengen Disziplinierungsmaßnahmen unterworfen werden.

Nach der französischen Revolution und mit der beginnenden Industrialisierung im 19. Jahrhundert haben sich die Verhältnisse grundlegend geändert. Wie ein Pariser Reiseführer aus dem Jahr 1863 zeigt, werden allein verkehrstechnisch die Distanzen aufgehoben, was ganz neue Dimensionen der Kommunikation, nicht nur im Sinne von Verkehrsverbindungen, ermöglicht:

Il existe au XIXe siècle une certaine agitation, un puissant besoin de mouvement, qui n'étaient pas connus de nos ancêtres; puis nous possédons, pour satisfaire ce besoin devenu chaque jour plus impérieux, une facilité admirable de communications, une promptitude vraiment féerique à franchir les distances. Partout on voyage avec rapidité, commodément, sans dangers probables et sans fatigues. Voyez toutes ces belles routes tracées jusque sur le sommet des plus hautes montagnes. À leur tour, les bateaux à vapeur nous conduisent aussi vite à Saint-Pétersbourg et à New York, que jadis les voituriers de Paris à Marseille, et les chemins de fer feront bientôt de tout un royaume une seule province, de l'Europe un

2 Open Access. (c) 2021 Michael Bernsen, publiziert von De Gruyter (c))BY-NC-ND Dieses Werk ist lizenziert unter der Creative Commons Attribution 4.0 Lizenz.

https://doi.org/10.1515/9783110758009-013 
seul pays, enfin, de notre planète entière, comme un seul et vaste domaine qu'on pourra désormais parcourir en l'espace de quelques mois. ${ }^{1}$

Die Räume der öffentlichen und quasi öffentlichen Konversation werden erheblich erweitert und große Gruppen der Bevölkerung sind nun in das Kommunikationsgeschehen involviert. Neben die privaten Konversationen im Freundeskreis, der Familie, der Nachbarschaft, treten Kommunikationen im öffentlichen Raum wie Cafés, Klubs, geschäftliche Treffen, Wahlveranstaltungen, Parlament u.a. ${ }^{2}$ Dabei verändern sich naturgemäß die normgebenden Instanzen dieser Gespräche. Setzten früher bei Hof und im Salon die gebildete oder machtvolle Einzelperson bzw. einzelne Gruppen mit entsprechenden gesellschaftlichen Verbindungen Maßstäbe einer ,bienséance', so werden nun in den erweiterten Räumen der Kommunikation Zeitungen und Bücher eines sich rasant entwickelnden Buchmarktes zur normgebenden Instanz. Für das 19. Jahrhundert hat der Soziologe und Philosoph am Collège de France, Gabriel Tarde, in seinem Buch L'Opinion et la foule (1901) die Entwicklung der Konversation analysiert und die zentrale Rolle der Presse sowie des Buchs herausgestellt:

La plus grande force qui régisse les conversations modernes, c'est le livre, c'est le journal. Avant le déluge des deux, rien n'était plus différent d'un bourg à l'autre, d'un pays à l'autre, que les sujets, le ton, l'allure des entretiens, ni de plus monotone, en chacun d'eux, d'un temps à l'autre. À présent, c'est l'inverse. La presse unifie et vivifie les conversations, les uniformise dans l'espace et les diversifie dans le temps. Tous les matins, les journaux servent à leur public la conversation de la journée. On peut être à peu près sûr, à chaque instant du sujet des entretiens entre hommes qui causent dans un cercle, dans un fumoir, dans une salle des Pas Perdus. ${ }^{3}$

Ganz neue Bevölkerungschichten kommunizieren miteinander. Dies verändert die Art der Kommunikation:

[...] par le changement des mœurs dans un sens démocratique, ce n'est pas seulement le nombre des interlocuteurs possibles qui s'accroît, c'est leur qualité qui varie. Les diverses couches sociales entrent plus librement en conversation; et, par l'émigration des champs

1 Paris-Touriste. Annuaire illustré des touristes et baigneurs, Paris 1863, S. 2.

2 Die grundlegenden Veränderungen seit dem 18. Jahrhundert hat Jürgen Habermas in seiner Habilitationsschrift beschrieben: Strukturwandel der Öffentlichkeit. Untersuchungen zu einer Kategorie der bürgerlichen Gesellschaft (Politica. 4), Neuwied/Berlin, Luchterhand, 1962; Neuauflage: (suhrkamp taschenbuch wissenschaft. 891), Frankfurt a. M., Suhrkamp, 1991.

3 Zitierte Ausgabe: (Collection Recherches politiques), Paris, Les Presses universitaires de France, 1989, S. 51; online: Chicoutimi, Québec, 2003: http://classiques.uqac.ca/classiques/ tarde_gabriel/opinion_et_la_foule/tarde_opinion_et_la_foule.pdf. (Die Seitenangaben im laufenden Text beziehen sich auf diese Ausgabe). 
aux villes, par l'urbanisation des campagnes mêmes, par l'élévation du niveau moyen de l'instruction générale, la nature des entretiens devient tout autre, de nouveaux sujets se substituent aux anciens. (Ebd.)

Anfänglich habe die Presse nur Themen aus den Räumen einer begrenzten öffentlichen Konversation aufgegriffen; nunmehr gebe sie jedoch die Sujets vor und forme eine öffentliche Meinung:

Les journaux ont commencé par exprimer l'opinion, l'opinion d'abord toute locale de groupes privilégiés, une cour, un parlement, une capitale, dont ils reproduisaient les commérages, les discussions, les discours; ils ont fini par diriger presque à leur gré et modeler l'opinion, en imposant aux discours et aux conversations la plupart de leurs sujets quotidiens. (S. $39 \mathrm{f}$.)

Man könne sich gar nicht vorstellen, in welchem Ausmaß das Buch und die Presse die Konversation beeinflusst habe:

On ne saura, on n'imaginera jamais à quel point le journal a transformé, enrichi à la fois et nivelé, unifié dans l'espace et diversifié dans le temps les conversations des individus, même de ceux qui ne lisent pas de journaux, mais qui, causant avec des lecteurs de journaux, sont forcés de suivre l'ornière de leurs pensées d'emprunt. Il suffit d'une plume pour mettre en mouvement des millions de langues. (S. 40)

Tarde begrüßt diese Entwicklung, insbesondere die uniformisierende Wirkung der Presse und des Buchs. Die Konversation im Ancien Régime war für ihn allein durch Hierarchien geprägt, so dass nach der vertikalen Ausrichtung des Gesprächs, das vor allem auf das Komplimentieren ausgerichtet war, eine echte, auf dem Austausch beruhende Konversation erst mit der Abschaffung der Ungleichheit entstehen konnte:

[...] on doit reconnaître que le compliment a été la relation unilatérale qui, en se mutualisant, à mesure que l'inégalité s'atténuait, est devenue la conversation [...](S. 46)

In Zeiten der Gleichheit der Bürger in der Demokratie verliert die Konversation laut Tarde mehr und mehr den Status eines Kampfes und nimmt die Konturen eines Austauschs an: „À travers les sinuosités capricieuses de ses divers courants, elle [= la conversation] tend à devenir de moins en moins une lutte et de plus en plus un échange d'idées.“ (S. 53). Die Konversation dient auf diese Weise dem Abbau von Egoismen und wird zum sozialen Kitt, der die Gesellschaft zusammenhält:

Au point de vue moral, elle [=la conversation] lutte continuellement, et avec succès le plus souvent, contre l'égoïsme, contre le penchant de la conduite à poursuivre des fins tout individuelles; elle trace et creuse, l'opposant à cette téléologie individuelle, une téléologie toute sociale [...] (S. 61) 
Insgesamt sieht er im Zeitungswesen eine Art öffentliches Gespräch, welches die einzelnen Meinungen zur öffentlichen Meinung verschmelzt:

En somme, le journal est [...] une conversation publique [... Il a commencé par n'être qu'un écho prolongé des causeries et des correspondances, il a fini par en être la source presque unique. [...] D’un télégramme privé adressé à son directeur, il fait une nouvelle à sensation d'une actualité intense [... I Il a achevé de la sorte le long travail séculaire que la conversation avait commencé, [...] le travail de fusion des opinions personnelles en opinions locales, de celles-ci en opion nationale et en opinion mondiale, l'unification grandiose de l'Esprit public. (S. 73)

Tarde entpuppt sich ganz als Vertreter eines für Frankreich typischen Egalitarismus, der seit der radikalen Abschaffung der Hierarchien des Ancien Régime in der Revolution bis heute vor allem das Justiz- und das Bildungswesen bestimmt. Schon Alexis de Tocqueville war in seinem Hauptwerk De la Démocratie en Amérique (1835/1840) vom Gedanken der Gleichheit in der amerikanischen Demokratie fasziniert, obgleich er davor warnte, dass ein Staat nicht die Gleichheit auf Kosten der Freiheit in den Mittelpunkt rücken und dadurch einem Egalitarismus verfallen sollte. Der Präsident der Dritten Republik am Ende des 19. Jahrhunderts, Jules Ferry, war der Meinung, die Gleichheit sei die Essenz und die legitime Basis der Gesellschaft, eine Position, die bis heute in Frankreich politisch verbreitet ist und rezente Kritiker dazu führt, dieses politische Konzept als eine der größten Pathologies politiques françaises zu beleuchten ${ }^{4}$.

Bemerkenswert an den Positionen Tardes' ist, dass er die uniformisierende Wirkung der Presse und des Buchs auf die Konversation als zivilisatorischen und sozialen Fortschritt gegenüber dem hierarchischen System der vorrevolutionären Zeit preist. Was nun aber die von ihm geschätzte Art und Weise des Sprechens in der Konversation angeht, hält er sich ganz an das Modell des Ancien Régime, ohne zu problematisieren, dass das indirekte Sprechen ein Produkt der vertikalen Hierarchien der Konversationsgesellschaften der Zeit vor der Revolution ist. Das Kriterium der ,politesse` der alten Gesellschaft sei zwar Ausdruck einer Ergebenheit gegenüber Höhergestellten; es sei jedoch derart zur ,habitude‘ geworden, dass es auch unter Bedingungen der Gleichstellung der Konversationsteilnehmer Anwendung finde:

Laisser parler l'interlocuteur est une marque de politesse à laquelle on ne se résout d'abord qu'en faveur d'un supérieur, sauf à la pratiquer à l'égard de tout le monde quand l'habitude en est prise. Cette habitude ne saurait donc se généraliser dans un pays que grâce à une assez longue discipline antérieure. Voilà pourquoi il convient, je crois, de faire procéder des conversations [...] les progrès de l'art de causer tel que nous le connaissons. (S. 48; Hervorhebung von Vf.)

4 Alain Duhamel, Les Pathologies politiques françaises, Paris, Plon, 2016. 
Auch unter den Bedingungen der Gleichstellung gelte es, die Konversation als jene tradierte Kunst („la conversation cultivée comme un art spécial“, S. 54) zu pflegen, für die Frankreich das universelle Modell („La France, [...] modèle universel“, S. 55) bereitgestellt habe. Tarde übernimmt die aristokratische Auffassung, die Rede eines Konversationsteilnehmers müsse eine hohe ästhetische Qualität aufweisen. Wollten die Teilnehmer der Gespräche im Ancien Régime damit ihre Freiheit und Individualität sichern, so werde das Kriterium nunmehr auf die zivilisierte Gesellschaft schlechthin übertragen:

Dans une société vraiment civilisée [...] il faut [...] que les moindres paroles, les moindres gestes, joignent toujours à leur caractère d'utilité, sans nulle affectation, un caractère de grâce ou de beauté propre. Il faut qu'il y ait des gestes „de style“ [... ] (S. 56)

Gegen Hippolyte Taine, der in seinem Hauptwerk Les origines de la France contemporaine (1877-1894) das Bedürfnis nach einer gehobenen Sprache und Rhetorik der Konversation als typisch für den Hof und die Salons angesehen hatte, argumentiert Tarde mit Tocqueville, der für egalitäre Gesellschaftssysteme die Notwendigkeit einer ,conversation fine' erkannt habe:

\begin{abstract}
Il [=ce besoin] se fait sentir encore, parmi nous, dans les oasis esthétiques de nos démocraties. Ne dirait-on pas, à lire Taine, que le goût de la conversation fine et de la vie de salon a été, non pas plus intense seulement sous l'ancien régime dans les classes supérieures, mais encore une singularité caractéristique et unique de la société française à cette phase de son développement? Là est l'erreur de cet esprit si pénétrant. [...] Tocqueville, avec plus de vérité, [...] explique la chose par l’influence du régime égalitaire. (Ebd.)
\end{abstract}

Die feine Konversation - hier kommt das indirekte Sprechen ins Spiel - war am Hof vor allem der Rücksichtnahme auf die Hierarchien (,un groupement hiérarchique d'hommes et de femmes“) geschuldet, um die unterschiedlichen ,amours propres“ in eine Balance zu bringen:

L’art de la conduite, en un tel milieu [...] suppose, avant tout, la distribution aisée, sûre, délicate, des nuances de respect dues à la diversité des mérites et des rangs; et le plaisir des amours-propres satisfaits par là dans une société éminemment hiérarchique est au moins aussi apprécié de tous que celui des idées échangées et accordées. (Ebd.)

In den Salons seien diese hierarchischen Unterschiede bereits nivelliert worden. Hier liegt für Tarde ein Vorläufer der gesellschaftlichen Gleichheit ${ }^{5}$ :

5 Als einen historisch vorbildlichen Salon beschreibt Tarde den des Baron d'Holbach, aus dem Diderot in seinem Brief an Sophie Volland von 1760 berichtet (S. oben Kap. 11.3, S. 226-230): „Le salon de Mme de Rambouillet était un salon littéraire et précieux, sans nulle liberté d'esprit - où 
[...] la vie de salon [...] tend à l'harmonie sociale [...] même en exprimant les distances des rangs, elle les atténue. [...] elle ne naît qu'entre égaux ou elle égalise, elle ne naît qu'entre semblables ou elle assimile. Seulement elle n'égalise et n'assimile qu'à la longue. (S. 57)

Aus dieser ,assimilation à la longue‘ entstehe eine Ästhetik der Konversation, die die ursprüngliche Schmeichelei aus hierarchischen Gründen durch eine unter Gleichen, die sich respektieren, ersetzt habe. Laut Tarde kann man im 19. Jahrhundert von einer Poetik der Konversation als einem quasi überzeitlichen ,code esthétique‘ sprechen:

$\mathrm{Au}$ point de vue esthétique, elle [= la conversation] engendre la politesse, par la flatterie unilatérale d'abord, puis mutualisée; elle tend à accorder les jugements du goût, y parvient à la longue et élabore ainsi un art poétique, un code esthétique, souverainement obéi à chaque époque [...] (S. 61)

Und diese Poetik der Konversation sehe das indirekte Sprechen als Regelfall vor: da man nicht immer davon ausgehen könne, auch unter den Bedingungen der Gleichheit der Gesprächsteilnehmer, dass die Komplimente aus Höflichkeit auf fruchtbaren Boden beim Gegenüber fallen. Man müsse demnach Konflikte durch eine allzu große Offenheit vermeiden. Das indirekte Sprechen, wenn auch in abgeschwächter Form, d. h. der sich abschwächenden Komplimente einer vertikalen Gesellschaft unter den Bedingungen der Gleichheit, werde zum Regelfall einer urbanen Konversation, die im Grunde und auf Dauer von einer grundsätzlichen Konfliktlosigkeit - „désintéressement“ in den Worten Tardes - geprägt ist.

De la conversation sont nés les compliments aussi bien que les injures. En causant, les hommes se sont aperçus que leur bonne opinion d'eux-mêmes n'était point partagée par autrui et réciproquement. L'illusion vaniteuse d'autrui, lorsqu'il s'agissait d'un égal, on pouvait la railler, la combattre durement en injuriant l'adversaire; encore l'expérience apprenait-elle à éviter les conflits provoqués par ces accès de franchise. Mais quand il s'agissait d'un supérieur, d'un maître, il était prudent de flatter cette chimère. De là les compliments qui, peu à peu, s'atténuant à la fois et se mutualisant, et se généralisant sous cette forme réciproque, sont devenus le fond de l'urbanité. Ils commencent toujours par être intéressés et ne deviennent désintéressés qu’à la longue. (S. 67; Hervorhebungen von Vf.)

il n'y avait d'un peu libre que la conversation amoureuse et galante (et encore)! - tandis que dans le salon d'Holbach on entendait, dit Morellet, la conversation la plus libre, la plus instructive et la plus animée qui fût jamais : quand je dis libre, j’entends en matière de philosophie, de religion et de gouvernement, car les plaisanteries libres dans un autre genre en étaient bannies'. [...] Le salon d'Holbach, comme celui d'Helvétius, comme ceux de toute la fin du XVIIIe siècle, rassemblaient des causeurs de toute classe et de toute nationalité, éclectisme qui n'eût pas été possible auparavant. Par la grande diversité d'origine des causeurs, comme par l'extrême variété et liberté de leurs sujets de conversation, ces salons différaient beaucoup des lieux de causerie antérieurs" (S. 52). 
All diese Veränderungen der Konversation gegenüber dem 18. Jahrhundert werden in den Romanen Honoré de Balzacs ausgiebig reflektiert. Balzac greift zudem einen weiteren Aspekt auf, der quasi als Gegenreaktion gegen den entstehenden Egalitarismus anzusehen ist: die Erfindung des modernen Subjekts. In dem Maße, in dem der Gedanke der Gleichheit nach der französischen Revolution Raum greift, in dem Maße denkt die Epoche darüber nach, wie sich das einzelne Individuum zur Geltung bringen und auszeichnen kann. Im Denken verhält sich das Individuum zu sich selbst und entwickelt ein Selbstbewusstsein. Je nach Fragestellung und philosophischer Position wird das Subjekt eher als ,Zugrundeliegendes“ (hypokeimenon) angesehen oder als ,Unterworfenes‘ (subiectum), welches sich den sozialen und gesellschaftlichen Gegebenheiten anpassen muss. Schaut man auf den Verstand und die Vernunft des Subjekts, steht der Aspekt der Erkenntnisfähigkeit und Urteilskraft im Mittelpunkt, schaut man auf den menschlichen Willen, steht die Frage nach dessen Freiheit im Raum. Aus einer historischen Perspektive betrachtet, stellt sich das Problem der Identität des Subjekts unter sich wandelnden Lebensbedingen. In all diesen Fällen hat das Subjekt ein massives Interesse daran, in der Kommunikation seine Rede zu verschleiern und die Kunst des Impliziten zu beherrschen: Entweder, um sich als einzigartiges Wesen von anderen Subjekten abzugrenzen (hypokeimenon). Dies ist die Grundlage der Stilisierung als Genie und seiner quasi unergründlichen Tiefendimension, wie sie in der Romantik allerseits anzutreffen ist. Oder die indirekte Art des Sprechens gilt der Dissimulation der Abhängigkeit von gesellschaftlichen Bedingungen und letztlichen Unfreiheit des Subjekts (subiectum). Die Postmoderne hat gezeigt, inwieweit die Vorstellung des beginnenden 19. Jahrhunderts, das Individuum zum machtvollen Subjekt des Wissens und der Geschichte zu machen, eine Fiktion ist. Allein die Abhängigkeit von der Sprache, die für die Erkenntnis der Lebensbedingungen die Raster und Floskeln bereitstellt und damit die Wahrnehmung filtert, zeugt von der Unfreiheit des Subjekts. Michel Foucault hat verdeutlicht, dass die romantische Vorstellung von der ,profondeur', der in der Tiefe des Subjekts zu entdeckenden Authentizität, Wahrheit und Identität, illusionär ist, da letztlich die Regeln der herrschenden Diskurse die sozialen Praktiken des Subjekts steuern und beherrschen. Gelangt man zu dieser Erkenntnis, ist die Kommunikation vor allem von der Vorstellung geprägt, die Abhängigkeit des ,subiectum zu verschleiern. Dies gilt insbesondere in einem Land, in dem die Strukturen weiterhin zentralistisch ausgerichtet sind.

In Balzacs Konzeption seiner Figuren kommen diese Aspekte zum Tragen. Balzac schreibt in der Zeit der Julimonarchie, in der das große Geld die Stadt Paris beherrscht. Die Kriterien der Beschleunigung des Lebens sowie des Utilitarismus sind in dieser Zeit klingende Münze. Seine Romane spielen jedoch zu einem großen Teil in der Epoche der vorangehenden Restauration, in der die Werte und 
Vorstellungen des Ancien Régime noch einmal vollumfänglich zu greifen sind. Wie Joachim Küpper gezeigt hat, entlehnt Balzac seine Figuren- und Konfliktkonstellationen dem zeitgenössischen Melodrama ${ }^{6}$. Dabei ist oftmals die weibliche Protagonistin die zentrale Figur der Comédie humaine: Ihr Wesen umgibt ein Geheimnis, welches an der Oberfläche nicht unmittelbar zu erkennen ist. Die Heldin ist entweder hoher Abstammung und lebt nach außen hin unter bescheidenen Lebensbedingungen oder sie ist niederer Abstammung und führt ein glänzendes Leben ${ }^{7}$. Die Tiefendimension der Figuren bei Balzac ist anders konzipiert als die der romantischen Heldinnen und Helden: In der Romantik hat das Geheimnis der Figur eine moralische Dimension. Ihre Leidenschaft richtet sich in der Regel eher ziellos auf eine unbestimmte Sehnsucht. Bei Balzac besteht das Geheimnis der Figuren in einer leidenschaftlichen Fixierung auf einen Gegenstand oder eine Person. Dem Autor geht es nicht mehr um die Tiefe der menschlichen Seele, sondern um die gesellschaftlichen Abgründe, die die Figuren zum Scheitern bringen. Die Balzacschen Helden sind Opfer der sozialen und gesellschaftlichen Untiefen. Ihr Geheimnis wird im Laufe der Handlung enthüllt ${ }^{8}$. Die realistische Erzählung Balzacs verweist auf die letztlich undurchdringliche Komplexität der Welt, an der die Helden scheitern ${ }^{9}$. Dies sind die Koordinaten, innerhalb derer Balzac ausgiebig über das Sprechen in der Konversation nachdenkt.

\subsection{Die Konversation der Figuren und die des Erzählers mit dem Leser: Les secrets de la princesse de Cadignan}

In seiner Abhandlung über die Histoire de la conversation stellt Godo die These auf, dass das 19. Jahrhundert aufgrund des für diese Epoche typischen „culte de l'utile“ keine Zeit mehr für die gepflegte Konversation lässt. Die Konversation sei zum Emblem einer seit der Revolution unwiederbringlich verlorenen Zeit geworden:

La conversation au XIX ${ }^{\mathrm{e}}$ siècle prend un tour que nous ne lui connaissions pas aux siècles précédents. Devenant, après la tourmente révolutionnaire, un point de ralliement de la

\footnotetext{
6 Balzac und der effet de réel. Eine Untersuchung anhand der Textstufen des Colonel Chabert und des Curé de Village (Beihefte zu Poetica. 17), Amsterdam, Grüner, 1986, S. 63. Zum Geheimnis der Balzacschen Romanfiguren vgl. auch Igor Sokologorsky, „Balzac et l'envers du monde“, L'Année balzacienne Bd. 10/2009, S. 315-345.
}

7 Vgl. S. $63 \mathrm{f}$.

$8 \mathrm{Vgl}$. S. $71 \mathrm{f}$.

9 Vgl. S. 75. 
noblesse exilée et défaite, elle est désormais liée à l'idée de nostalgie et apparaît comme l'emblème d'un temps à jamais enfui, concentrant toutes les amertumes, toutes les frustrations et toutes les insatisfactions de ceux qui ne se sentent pas en phase avec ce XIX ${ }^{\mathrm{e}}$ siècle qui commence à prôner le culte de l'utile. ${ }^{10}$

Godo beruft sich auf den Artikel „Conversation“ des Grand Larousse du XIXe siècle, der feststellt, dass die Kunst der Konversation eine mittlerweile vergessene Kunst sei, da die Politik und der Einfluss der Presse die aus der bedeutungslosen Aristokratie stammende Leichtigkeit und Grazie verdrängt habe:

Sous la République et sous l'Empire, on avait bien autre chose à faire qu'à causer. Lorsque, dans la France pacifiée, quelques salons s'ouvrirent, il se trouva que la conversation était un art oublié, et que son règne était fini. Les esprits avaient perdu cette grâce, cette légèreté, cette frivolité même, qui ne sauraient se trouver que dans une aristocratie. Les discussions politiques avaient remplacé les conversations galantes, philosophiques et artistiques, et l'influence croissante de la presse vint bientôt diminuer la part de la causerie des salons. ${ }^{11}$

Die Kunst der Konversation, des Impliziten und der Verstellung sei zu sehr an die Klassik und ihre Künstlichkeit und Oberflächlichkeit gebunden, um die an $\mathrm{Au}-$ thentizität und Wahrheit der an der inneren Tiefe des Subjekts interessierte Romantik beeindrucken zu können. Wenn Balzac sich daher in mehreren Novellen und Romanen als faszinierter Anhänger der Konversation zu erkennen gebe, dann aus einer nostalgischen Bewunderung der Aristokratie heraus und aus der Ablehnung der zeitgenössischen Unterwerfung unter den Utilitarismus:

La conversation, en tant que libre et désintéressée, se révèle une manière anodine et néanmoins radicale de se révolter contre la pensée dominante. C’est une révolte élégante, spirituelle, un déni poli et cependant ferme aux injonctions malséantes du temps présent. [...] la conversation se pense sur le mode de l'antagonisme - il s'agit désormais de se distinguer de la pensée commune que symbolise le sérieux positif de l'article de journal et du discours politique. La conversation devient alors l'instrument d'affirmation et de différenciation d'une élite pratiquant la misanthropie collective et manifestant par elle son désaccord de fonds vis-a-vis de cette société bourgeoise. ${ }^{12}$

Nun ist Balzac zweifellos von den vergangenen Epochen der aristokratischen Gesellschaft fasziniert. Er ist allerdings nicht in erster Linie Nostalgiker. Und er ist auch kein Romantiker. Er analysiert die sozialen Abgründe seiner Zeit und greift

10 S. 259.

11 Artikel „Conversation”, in: Nouveau Larousse illustré. Dictionnaire universel encyclopédique, hrsg. von Claude Augé, 7 Bde., Paris, Librairie Larousse, 1897-1904, Bd. 3, S. 252. Vgl. Godo, Une Histoire, S. 260.

12 S. 295. 
deshalb auf die Konversation zurück, weil ein wesentlicher Zweck der Konversationen der Salons die Ergründung der Geheimnisse der Gesprächspartner ist und sich dies mit der Konzeption seiner Romanfiguren gut vereinbaren lässt. Anders als Godo meint, tragen seine literarischen Reflexionen zur Konversation der erheblich komplexeren Gemengelage Rechnung, wie sie Tarde beschreibt. Vorgeführt und zugleich durch den Erzähler reflektiert werden Gesprächsabläufe der Zeit in einer längeren Novelle, die in der Balzacliteratur bislang kaum Beachtung gefunden hat: Les Secrets de la princesse de Cadignan (1839) ${ }^{13}$.

Der männliche Protagonist dieser Novelle ist der aus dem ,Cénacle‘ der Illusions perdus (1835), dem Gesprächskreis einiger Künstler, bekannte Schriftsteller Daniel d'Arthez, der inzwischen nicht nur literarische Anerkennung erlangt, sondern es auch zu einem Adelstitel gebracht hat und darüber hinaus durch eine Erbschaft zu einem gewissen Reichtum gekommen ist. Dieser junge und unerfahrene Autor trifft auf die weibliche Protagonistin, die 36 Jahre alte Duchesse de Maufrigneuse, Princesse de Cadignan, vor ihrer Heirat Diane D’Uxelles, die in ihrer Vergangenheit zahlreiche Männer gekannt und deren Vermögen durchgebracht hat. Die Princesse verliebt sich in den jungen Schriftsteller und verführt ihn, indem sie ihm ihre turbulente Lebensgeschichte so erzählt, dass sie als Suche nach der ewigen und wahren Liebe einer unschuldigen Frau erscheint. Bei einem Essen mit einigen seiner Freunde versuchen diese d'Arthez ein letztes Mal aufzuklären und zu warnen. Dieser ergreift allerdings vehement Partei für die Princesse, indem er zu erkennen gibt, dass er all ihre Taten kennt, die Princesse aber dennoch für verehrungswürdig hält.

Der Roman besteht im Wesentlichen aus einer Aneinanderreihung mehrerer Gespräche, in deren Zentrum die Unterhaltungen der Princesse mit Daniel d'Arthez stehen. Ein deklariertes Ziel des Romans ist es, die Kunst des Impliziten ins Bild zu setzen, mit der die Princesse den jungen Schriftsteller von ihrer Unschuld und Aufrichtigkeit zu überzeugen sucht. Diese Strategie wird durch den Erzähler begleitend reflektiert. Es finden auf diese Weise zwei Unterhaltungen gleichzeitig statt: Die der Figuren untereinander sowie die des Erzählers mit seinen Lesern, welche vom Erzähler fortwährend angeredet bzw. befragt werden.

Die Unterhaltungen zwischen d'Arthez und der Princesse haben den Charakter eines ,aveu', in dessen Verlauf die Protagonistin dem jungen Schriftsteller ihr

13 Zur Textgeschichte dieser Novelle, der ursprünglichen Titelgebung Une princesse parisienne, sowie zum historischen Fall der Princesse de Cadignan, auf die sich die Novelle bezieht vgl. die „Introduction“ von Anne-Marie Meininger, in: Honoré de Balzac, La Comédie humaine, 12 Bde. (Bibliothèque de la Pléiade. 26. 27. 30. 31. 32. 35. 38. 39. 41. 42. 141. 292), hrsg. von Pierre-Georges Castex u. a., Paris, Gallimard, 1976-1981, Bd. 6, S. 937-1005, hier: S. 939-947 sowie die "Histoire du texte", S. $1508 \mathrm{f}$. 
schreckliches Geheimnis preisgibt. Vorgewarnt durch seine Freunde, auf eine der berüchtigtsten Damen der Pariser Gesellschaft zu treffen („la plus monstrueuse Parisienne, la plus habile coquette, la plus enivrante courtisane du monde“"14), geht d’Arthez mit großer Neugier in diese Gespräche. Die Princesse ist auf den jungen Autor aufmerksam geworden, weil er ihr als einer jener außergewöhnlichen Menschen („hommes rares“, S. 962) erscheint, der gerade wegen seiner Widersprüche ihr Interesse weckt und ihre Geschichte glaubhaft finden könnte: Wenig erfahren in Liebesdingen sowie im Auftritt in der großen Gesellschaft, hat er sich eine kindliche Naivität und Unschuld bewahrt, obgleich er doch als erfolgreicher Autor einer der schärfsten Beobachter der Pariser Gesellschaft ist („[...] il reste l'enfant le plus candide, en se montrant l'observateur le plus instruit [... ]“, S. 963). Der Erzähler rechtfertigt diese Widersprüche als nur scheinbare:

Ce contraste, en apparence impossible, est très explicable pour ceux qui ont pu mesurer la profondeur qui sépare les facultés des sentiments: les unes procèdent de la tête et les autres du cœur. On peut être un grand homme et un méchant, comme on peut être un sot et un amant sublime. D’Arthez est un de ces êtres privilégiés chez lesquels la finesse de l'esprit, l'étendue des qualités du cerveau, n'excluent ni la force ni la grandeur des sentiments. Il est, par un rare privilège, homme d'action et homme de pensée tout à la fois. (S. 963)

Dieser unerfahrene Schriftsteller wird im Lauf der Gespräche die quasi religiösen Dimensionen der Liebe kennenlernen. Sie werden für ihn zur Begegnung mit einer engelgleichen Frau, einer ,femme ange،, wie man sie seit Dantes Vita nova in Gestalt der Beatrice kennt, die den Liebenden zu den göttlichen Dimensionen der Liebe führt:

L'une des gloires de la Société c'est [...] d'avoir [...] inventé l'amour, la plus belle religion humaine. D’Arthez ne savait rien des charmantes délicatesses de langage, rien des preuves d'affection incessamment données par l'âme et l'esprit, rien de ces désirs ennoblis par les manières, rien de ces formes angéliques prêtées aux choses les plus grossières par les femmes comme il faut. Il connaissait peut-être la femme, mais il ignorait la divinité. (S. 964 f.)

Damit stellt der Roman auch die Frage in den Raum, ob diese göttlichen Attribute der Liebe überhaupt existieren, die in den Vorstellungen der Romantik so intensiv gepriesen werden und deren Tiefendimensionen nur das Genie auszuloten vermag.

Von ganz besonderen Ausmaßen sind die Geheimnisse der Princesse, die ebenfalls von Widersprüchen geprägt ist. In diese Geheimnisse lässt sie den

14 Zitierte Ausgabe s. Anm. 13, S. 967. Die Seitenangaben im laufenden Text beziehen sich auf diese Ausgabe. 
jungen d'Arthez und mit ihm den Leser nach und nach eindringen: Als junges Mädchen ist Diane d'Uxelles im Alter von 17 Jahren an den Duc de Maufrigneuse verheiratet worden. Das Geständnis, dessen Enthüllung der Erzähler sensationserheischend zelebriert, entbirgt eines jener „drame[s] horrible[s]“ (S. 979), die sich in der Pariser Gesellschaft ereignen und laut Erzähler jede literarische Erfindung in den Schatten stellen ${ }^{15}$ : Der Duc de Maufrigneuse, Prince de Cadignan, ist der Liebhaber der Mutter von Diane. Die Heirat mit der Tochter wird inszeniert, um die Geburt des unehelichen Sohnes der beiden zu kaschieren. Die junge Diane muss das uneheliche Kind ihrer Mutter mit dem Herzog als ihren eigenen Sohn anerkennen und aufziehen. Ihr Vermögen dient zudem der finanziellen Rettung des völlig verschuldeten Herzogs. Derart in die Untiefen der Pariser Gesellschaft abgestürzt, sucht Diane d'Uxelles zahlreiche Affären und verbringt ihr Leben, ohne jemals wirklich geliebt zu haben ${ }^{16}$. Die Ruinierung ihrer Liebhaber, die sie dabei in Kauf nimmt, sieht sie als Rache an ihrer misslichen Situation ${ }^{17}$.

Das Geständnis dieses Geheimnisses wird nun von der Protagonistin dazu benutzt, um mit allen Mitteln der Kunst des Impliziten, den jungen d'Arthez von ihrer Unschuld und Reinheit in Liebesfragen zu überzeugen und ihn auf diese Weise zu verführen. Es geht darum, ihn die Wahrheit glauben zu lassen (,faire croire à une vérité“, S. 959). Dieses Ziel erreicht die Princesse insbesondere mit der theatralischen Inszenierung der Gesprächssituation, ihrer persönlichen Aufmachung, ihrer Körpersprache sowie ihrer Rede. Dabei bleibt sie zu jeder Zeit völlig undurchdringlich und kann so je nach Bedarf diverse Rollen annehmen:

Il était impossible au physionomiste le plus habile d'imaginer des calculs et de la décision sous cette inouïe délicatesse de traits. Il est des visages de femmes qui trompent la science et déroutent l'observation par leur calme et par leur finesse; [...] La princesse est une de ces femmes impénétrables, elle peut se faire ce qu'elle veut être: folâtre, enfant, innocente à désespérer; ou fine, sérieuse et profonde à donner de l'inquiétude. (S. 969)

Beim ersten Gespräch mit d'Arthez kommt sie frühzeitig, wählt ihren Sitzplatz aus und nimmt eine Position ein, die für die Verwirklichung ihrer Intention günstig ist:

15 „Ah! vous ne savez pas qu'en inventant vos drames, ils sont surpassés par ceux qui se jouent dans les familles en apparence les plus unies. Vous ignorez l'étendue de certaines infortunes dorées.” - sagt die Princesse zu d'Arthez. (S. 987)

16 „Pour m'étourdir, pour oublier la vie réelle par une vie fantastique, j’ai brillé, j’ai donné des fêtes, j'ai fait la princesse, et j'ai fait des dettes.” - heißt es. (S. 994)

17 „Préoccupée de ma vengeance [...]“ (S. 992). 


\begin{abstract}
Elle vint chez la marquise avec l'intention d'être une femme douce et simple à qui la vie était connue par ses déceptions seulement, une femme pleine d'âme et calomniée, mais résignée, enfin un ange meurtri. Elle arriva de bonne heure, afin de se trouver posée sur la causeuse, au coin du feu, près de madame d'Espard, comme elle voulait être vue, dans une de ces attitudes où la science est cachée sous un naturel exquis, une de ces poses étudiées, cherchées [...] (Ebd.)
\end{abstract}

In ihrer Körpersprache äußert sich - auf eine unaussprechliche Art und Weise, wie der Erzähler vermerkt - das ganze versteckte Unglück ihres Lebens:

[...] la princesse hocha la tête en agitant ses belles boucles blondes pleines de bruyères par un geste sublime. Ce qu'elle exprimait de doutes désolants, de misères cachées, est indicible." (S. 971)

Und wenn sie spricht, verfehlen ihre Worte nie das Ziel:

Ces paroles, soufflées à l'oreille de l'écouteur de manière à être dérobées à la connaissance des convives, et avec un accent digne de la plus habile comédienne, devaient aller au cœur; aussi atteignirent-elles à celui de d'Arthez. (S. 972)

Der Erzähler fasst diese Kunst des Impliziten, die Macht der indirekten Kommunikation zusammen, wenn er der Princesse bescheinigt, eine letzten Endes unerklärliche Aura und damit Tiefendimension zum Ausdruck zu bringen:

Les femmes savent donner à leurs paroles une sainteté particulière, elles leur communiquent je ne sais quoi de vibrant qui étend le sens des idées et leur prête de la profondeur; si plus tard leur auditeur charmé ne se rend pas compte de ce qu'elles ont dit, le but a été complètement atteint, ce qui est le propre de l'éloquence. (S. 973)

Die Princessin wird in den Augen des jungen Schriftstellers quasi zur Metonymie der Dichtung: „Ainsi la princesse avait aux yeux de d'Arthez un grand charme, elle était entourée d'une auréole de poésie“ (S. 973). Sie wird dies allerdings nicht durch das literarische Gespräch und ihre Kenntnisse in der Literatur. In ihrer quasi professionellen Vorbereitung der Verführung des jungen Autors hat sich die Princesse sogar zuvor in dessen Werke eingelesen. Aber es ist nicht dieses Terrain, auf dem ihre Stärke liegt und auf dem sie den unerfahrenen d'Arthez von der Reinheit ihrer Liebe überzeugt. Ihrer zeitweilig zur Schau gestellte literarische Gelehrsamkeit bringt die beiden eher auseinander, so dass sie schnell auf den ihr eigenen Schauplatz zurückkehrt: „Ces conversations éloignaient Diane du but, elle essaya de se retrouver sur le terrain des confidences [...]“ (S. 984) Es ist das Spiel der ,causerie facile', der scheinbar belanglosen „émouvantes irrésolutions“ (S. 985), der „délicieux enfantillages“ (ebd.) der indirekten Kommunikation des Salon, welches ihr eine „auréole de poésie“ verleiht und sie gleichsam zu einer großen Künstlerin werden lässt: 
Elle ressemblait à un grand artiste se complaisant dans les lignes indécises d'une ébauche, sûr d'achever dans une heure d'inspiration le chef-d'œuvre encore flottant dans les limbes de l'enfantement. (S. 985)

Balzacs Roman Les secrets de la princesse de Cadignan, reflektiert auf diese Weise die Macht der ,causerie‘ und deren Übergang in die Literatur im 19. Jahrhundert. Die indirekte Kommunikation der Salons ist für ihn eine literarische Leistung, die unter den Verhältnissen des 19. Jahrhunderts, in denen der Utilitarismus und die Beschleunigung des öffentlichen Lebens von der Literatur und der Kunst weitergeführt werden. Die Kunst des Impliziten im mündlichen Gespräch wie in der Literatur vermag die größten Widersprüche als Wahrheit zu plausibilisieren. Die im Roman reflektierte Frage lautet: Kann man einem literarischen Genie wie Daniel d'Arthez vermitteln, dass die größten Widersprüche des Lebens existieren, im Fall der Princesse de Cadignan, dass eine Frau, die sich die schlimmsten moralischen Verfehlungen geleistet hat, im Innern ihres Herzens unschuldig ist:

Innocente comme un enfant, je passais pour une femme perverse, pour la plus mauvaise femme du monde [...] (S. 992)

Und es schließt sich die Frage an, ob man jemanden bedingungslos lieben kann, der selbst schonungslos all seine Verfehlungen aufgedeckt hat:

D’Arthez rebaisa la main de cette sainte femme qui, après lui avoir servi une mère hachée en morceaux, avait fait du prince de Cadignan, que vous connaissez, un Othello à triple garde, se mettait elle-même en capilotade et se donnait des torts, afin de se donner aux yeux du candide écrivain cette virginité que la plus niaise des femmes essaie d'offrir à tout prix à son amant. (S. 994)

Die Antwort des männlichen Protagonisten fällt positiv aus, und zwar deshalb, weil die Princesse erfolgreich alle Mittel der Täuschung angewandt hat, um letztlich ihre Wahrheit ans Licht zu bringen. Die Rede der Princesse wird auf eine Ebene mit der Literatur gehoben. Wie die Rede ist auch die Literatur Täuschung, ein indirektes Sprechen über die angebliche Wahrheit, ein ,effet de réel', wie ihn Roland Barthes am Beispiel der Comédie humaine analysiert hat. Balzac beleuchtet die ,causerie' der beiden Protagonisten jedoch noch aus einer anderen Pespektive, indem er den Erzähler in eine Konversation mit dem Leser eintreten lässt. Und diese Unterhaltung wird ganz anders als die der Princesse mit d'Arthez mit einer geradezu schonungslosen Klarheit geführt.

Der Erzähler lässt keine Gelegenheit aus, das Spiel der Princesse als moralisch fragwürdig zu entlarven. Diese Fragwürdigkeit wird durch seine Einlassungen von Beginn an herausgestellt: „,...] cette illustre égoïste songea que le sentiment maternel poussé à l'extrême deviendrait pour sa vie passée une absolution 
confirmée par les gens sensibles [...]“(S. 952) Ihre scheinbar unschuldigen, indirekten körpersprachlichen Äußerungen (,[...] en tournant son doux et noble visage vers d'Arthez par un mouvement plein de confusion pudique“, S. 972), tut er unter dem Stichwort ,komödiantisches Talent' ab, gegen die das Drama eines Tartuffe eine Petitesse sei (,[...] auprès desquels le drame de Tartuffe est une vétille [...]“, S. 979):

Une des plus savantes manœuvres de ces comédiennes est de voiler leurs manières quand les mots sont trop expressifs, et de faire parler les yeux quand le discours est restreint. Ces habiles dissonances, glissées dans la musique de leur amour faux ou vrai, produisent d'invincibles séductions. (S. 972)

Auch die vom Erzähler verwendete Jagdmetaphorik ist nicht gerade dazu angetan, den Leser von der romantischen Idee der unschuldigen und reinen Liebe der Princesse zu überzeugen:

Ce caquetage fut sifflé d'une voix si doucement moqueuse, si mignonne, avec des mouvements de tête si coquets, que d'Arthez, à qui ce genre de femme était totalement inconnu, restait exactement comme la perdrix charmée par le chien de chasse. (S. 981)

Die Gespräche der Princcesse mit d'Arthez sind für den Erzähler ein Spiel (,ne fut ce que pour le jouer“, S. 977), mit dem Ziel, den jungen Autor durch die Manege zu führen (Ce manège, froidement convenu mais divinement joué“, S. 985) und ihn zu beherrschen („le plaisir de [...] maîtriser“, S. 977).

So wie die Princesse d'Arthez von ihrer Unschuld und Reinheit überzeugt, so entlarvt der Erzähler im Gespräch mit dem Leser das Vorgehen der Duchesse als durchkalkuliertes Lügengebäude: „[...] comptez qu'elle s'était préparée à cette heure de comique mensonge avec un art inouï dans sa toilette [... ]“ (S. 989) Wie in einer Salonkonversation äußert der Erzähler dem Leser gegenüber seine private Meinung und stellt ihm immer wieder Fragen:

Trompeuse ou vraie, elle enivrait Daniel. S'il est permis de risquer une opinion individuelle, avouons qu'il serait délicieux d'être ainsi trompé longtemps. Certes, souvent Talma, sur la scène, a été fort au-dessus de la nature. Mais la princesse de Cadignan n'est-elle pas la plus grande comédienne de ce temps? (S. 989)

Auf diese Weise präsentiert der Roman zwei unterschiedliche Unterhaltungen: In der einen lässt sich das junge schriftstellerische Genie Daniel d'Arthez mit all seiner Beobachtungsgabe, Feinsinnigkeit und unschuldigen Naivität allein durch die Erzeugung einer scheinbaren Wahrheit von der romantischen Idee einer Tiefendimension der Liebe überzeugen, und das in voller Kenntnis der moralisch fragwürdigen Taten der Princesse: 
D’Arthez était abasourdi. [...] Cette atroce élégie, forgée dans l'arsenal du mensonge et trempée aux eaux du Styx parisien, avait été dite avec l'accent inimitable du vrai. (S. 995)

In der anderen überzeugt der Erzähler den Leser, dass es aus den Untiefen der sozialen Abgründe, in die die Protagonistin schon frühzeitig gefallen ist, kein Entrinnen gibt. Sie ist, so die Quintessenz dieser Unterhaltung, keine ,femme ange‘, sondern ein durch und durch maliziöses Wesen. Zugleich werden die Dimensionen der romantischen ,profondeur als Täuschungen indirekten Sprechens entlarvt:

Ce fut le dernier coup, et le pauvre d'Arthez n'y tint pas : il se mit à genoux, il fourra sa tête dans les mains de la princesse, et il y pleura, il y versa de ces larmes douces que répandraient les anges, si les anges pleuraient. Comme Daniel avait la tête là, Mme de Cadignan put laisser errer sur ses lèvres un malicieux sourire de triomphe, un sourire qu'auraient les singes en faisant un tour supérieur, si les singes riaient. „Ah! je le tiens“, pensa-t-elle; et, elle le tenait bien en effet. (S. 995 f.)

Die Darstellung des zeitgenössischen gesellschaftlichen Lebens durch den „sécrétaire de la société française“, wie sich Balzac im „Avant-Propos“ seiner Comédie humaine selbst stilisiert ${ }^{18}$, zielt auf die Erfassung und Darstellung der totalen Gegensätze. Und so treffen in den Secrets de la princesse de Cadignan zwei völlig unterschiedliche Konversationen mit zwei völlig verschiedenen Stoßrichtungen aufeinander, die auch zwei Weisen des Sprechens repräsentieren: die implizite der Princesse und die schonungslos offene des Erzählers. Die Frage, welche der beiden Konversationen die wahre und richtige ist, lässt der Erzähler jedoch am Ende offen ${ }^{19}$ :

La princesse a hérité de sa mère quelque fortune, elle passe tous les étés à Genève dans une villa avec le grand écrivain, et revient pour quelques mois d'hiver à Paris. D’Arthez ne se montre qu'à la Chambre, et ses publications sont devenues excessivement rares. Est-ce un dénouement? Oui, pour les gens d'esprit; non, pour ceux qui veulent tout savoir. (S. 1004 f.)

$\mathrm{Zu}$ Beginn des 19. Jahrhunderts stellt sich somit die entscheidende Frage: Steht die Konversation als Kunst des Impliziten höher im Kurs oder die der Klarheit

18 In: Balzac, La Comédie humaine, Bd. 1, S. 7-20, hier: S. 11.

19 Zur Unterhaltung des Erzählers mit dem Leser in den Secrets de la princesse de Cadignan, ohne allerdings genau zu benennen, wohin diese Unterhaltung führen soll, vgl. Laelia Véron, „Jeu de mots et double communication dans l'oeuvre littéraire: l'exemple de la Comédie humaine de Balzac“, in: Esme Winter-Froemel/Angelika Zirker (Hrsg.), Enjeux du Jeux de mots. Perspectives linguistiques et littéraires (The Dynamics of Wordplay. 2), Berlin/Boston, De Gruyter, 2015, S. 93-111, hier: S. $106 \mathrm{f}$. 
und schonungslosen Offenheit? Die der „gens d'esprit“ oder die derjenigen, „qui veulent tout savoir“. Dass sich der Autor Balzac eher auf der Seite der ,gens d'esprit' wähnt, zeigt auch die Novelle Une conversation entre onze heures et minuit, die sich ebenfalls mit dem Thema der ,causerie‘ befasst.

\subsection{Konversation und das Implizite: Une conversation entre onze heures et minuit}

In der Novelle Une conversation entre onze heures et minuit, zuerst 1832 in den Contes bruns, 1844 erneut unter dem Titel Échantillon de causerie française veröffentlicht, bekräftigt Balzac diese Vermutung, wenn er den Erzähler zu Beginn sagen lässt, dass das mündliche Erzählen am Beginn der Literatur steht und dass die ,causerie‘ der Salons die perfekteste Form der Mündlichkeit sei, weil hier die Kunst des Impliziten ihre höchste Vollendung erfährt. Der Erzähler berichtet von einem Salon in Paris, bei dem Künstler, Dichter, Staatsmänner, Wissenschaftler, Damen der Gesellschaft und zahlreiche weitere Gruppen aufeinandertreffen. Dieser Salon ist, so der Erzähler, ein Asyl des tradierten „esprit français“. Und dieser Geist, den ja auch schon Mme de Staël beschrieben hatte ${ }^{20}$, zeichnet sich durch eine gewisse Tiefe aus: „Ce salon est le dernier asile où se soit réfugiél'esprit français d'autrefois, avec sa profondeur cachée, ses mille détours, sa politesse exquise.“21 Hier werden spontan Geschichten erzählt, ohne dass man darin gleich literarische Dramen oder ganze Bücher erkennen muss:

Là vous trouverez encore quelque spontanéité dans les cœurs, de l’abandon, de la générosité dans les idées. Nul ne pense à garder sa pensée pour un drame, ne voit des livres dans un récit. (Ebd.)

Die ,causerie، ist für den Erzähler die höchste Kunst des Impliziten, des indirekten Sprechens. Die Teilnehmer an solchen Konversationen hätten eine regelrechte künstlerische Ader:

20 Vgl. dazu oben, Kap. 5.1, S. 77.

21 Hier zitiert nach der Version von 1832. Die spätere Version wurde von Balzac mehrfach gekürzt, da er einzelne Geschichten aus der Novelle bereits anderweit verarbeitet hatte. Zitierte Ausgabe: http://www.bouquineux.com/index.php?telecharger=254\&Balzac-Une_conversation_ entre_onze_heures_et_minuit, S. 1. Die Seitengaben im laufenden Text beziehen sich auf diese Ausgabe. Zur Textgeschichte der Erzählung Echantillon de causerie française vgl. auch den Kommentar des Herausgebers Roger Pierrot der Comédie humaine in der Bibliothèque de la Pléiade, Bd. 12, S. 1015-1017. 
Ingénieuses réparties, observations fines, railleries excellentes, peintures dessinées avec une netteté brillante, pétillèrent et se pressèrent sans apprêt, se prodiguèrent sans dédain comme sans recherche, mais furent délicieusement senties, délicatement savourées. Les gens du monde se firent surtout remarquer par une grâce, par une verve tout artistiques. (Ebd.)

Als Zentrum dieses „esprit particulier“ gilt dem Erzähler Paris, die Hauptstadt der raffinierten indirekten Kommunikation und damit die Hauptstadt des guten Geschmacks, in der die Teilnehmer eines Gesprächs mit einem einzigen Wort ihre ganze Erfahrung zum Ausdruck bringen und ihre geistvolle Haltung in einem einzigen Merkmal ihrer Einlassung kondensieren können:

Vous trouverez ailleurs, en Europe, d'élégantes manières, de la cordialité, de la bonhomie, de la science; mais [...] Paris, capitale du goût, connaît seul cette science qui change une conversation en une joute, où chaque nature d'esprit se condense par un trait, où chacun dit sa phrase et jette son expérience dans un mot, où tout le monde s'amuse, se délasse et s'exerce. (Ebd.)

Konversationen dieser Art sind - so heißt es weiter - Ausdruck jener Oralität, die einen guten Schauspieler oder Erzähler ausmachen und den Zuhörer gleichsam verzaubern:

Jamais le phénomène oral qui, bien étudié, bien manié, fait la puissance de l'acteur et du conteur, ne m’avait si complètement ensorcelé [...] (S. 2)

Gleich bei der ersten Geschichte, die in diesem illustren Kreis erzählt wird, zeigt sich in vollem Umfang das, was Balzac fasziniert: Es ist vor allem das Implizite der Konversation, die sich über den erzählten Kasus entspinnt und die ganz unerwartete Verlaufsformen annimmt. Es ist jener letztlich unerklärbare Fluss der Gedanken, der dem Erzählten immer neue Wendungen gibt und neue Konnotationen freisetzt. Durch die Ideen der Gesprächsteilnehmer, ihre Einlassungen in Form von ergänzenden Erzählungen, historischen Erklärungen oder geistreichen Beurteilungen, werden solche Konnotationen freigesetzt:

[...] dans ce salon et dans quelques autres encore, se rencontre l'esprit particulier qui donne à toutes ces qualités sociales un agréable et capricieux ensemble, je ne sais quelle allure fluviale qui fait facilement serpenter cette profusion de pensées, de formules, de contes, de documents historiques. (S. 1)

Den Verlauf der ersten Erzählung im Gespräch und die Konnotationen, die er freisetzt, lohnt es sich zu verfolgen.

Ein alter General der napoleonischen Armee in Spanien weiß eine besonders sensationelle Geschichte zu erzählen: Ein italienischer Soldat namens Bianchi, 
der unbedingt 1000 Ecus benötigt, die er einer Dame versprochen hat, wettet mit einem ebenfalls italienischen Kameraden, dass er für die erforderliche Summe einem in geringer Entfernung postierten spanischen Wachposten das Herz herausschneiden und es nach der Zubereitung im Kochkessel über dem Lagerfeuer verspeisen werde. Als Gegenleistung, sollte er scheitern, bietet er seine beiden Ohren an. Die Geschichte nimmt ihren Verlauf wie angekündigt.

Balzac geht es nicht darum, im Vorgriff auf die ,contes cruels' der zweiten Hälfte des 19. Jahrhunderts eine kannibalische Geschichte zu erzählen; es geht ihm um die sich im Anschluss an die Geschichte entwickelnde Diskussion. Der Offizier schließt nämlich eine historische Erklärung an: In Italien seien nach einer alten Sitte Findelkinder unter dem Namen Bianchi im ,hôpital de Como“ (S. 5) zusammengetrieben und untergebracht worden. Diese „mauvais sujets“ (ebd.) habe Napoleon nach Elba deportieren lassen und sie später in einer italienischen Legion in seine Armee unter dem Kommando eines korsischen Colonels integriert. Die Truppe wurde wegen ihrer Tollkühnheit („des actions d'éclat“, ebd.), wegen ihrer Übergriffe aber auch wegen ihres Corpsgeistes und ihrer Kameradschaft berühmt-berüchtigt. Eine Dame wirft in die Diskussion ein, Napoleon habe „sehr philosophische Ideen“ gehabt („Napoléon avait bien des idées philosophiques!“, S. 6), denn man müsse schon gedanklich tief in die menschliche Natur eindringen, um herauszufinden, wie man aus solchen Übeltätern Helden mache. Und einer der „großen Dichter“ („grands poètes“, ebd.) der Zeit, dessen Name nicht genannt wird, schließt mit theatralischer Geste ein Loblied auf den Kaiser an, dessen Genialität es vermocht habe, die Zivilisation überall hin zu bringen:

Qui pourra jamais expliquer, peindre ou comprendre Napoléon!... Un homme qu'on représente les bras croisés, et qui a tout fait; qui a été le plus beau pouvoir connu, le pouvoir le plus concentré, le plus mordant, le plus acide de tous les pouvoirs; singulier génie, qui a promené partout la civilisation [...] (Ebd.)

Und dieses Eulogium nutzt der „grand poète” zu einem weiteren Loblied auf die Größe Frankreichs als dem maßgeblichen Land Europas: „N’a-t-il pas fait de l’Europe la France ?" (Ebd.)

Betrachtet man nun den Gedankenfluss, die „allure fluviale“, jenes „faire serpenter“ der Verbreitung der Ideen (,profusions des pensées“), die dieser Gesprächsverlauf impliziert und dadurch beim Leser Konnotationen freisetzt, dann ergibt sich eine erstaunliche vertikale Entwicklung: Die Tat Bianchis greift zunächst nur das in Frankreich verbreitete Klischee vom affektbesessenen und nicht immer zivilisierten Italiener auf: „Avanti, avanti, signori ladroni, cavalieri ladri [...]“ (S. 5) hatte jemand nach der Präsentation der Geschichte in die Debatte eingeworfen. Der Italiener als Kannibale fällt allerdings auf die französische Zivilisation zurück, da besagter Bianchi ja immerhin Soldat der französischen 
Armee ist. Kriegszeiten sind Zeiten der Barbarei, und dies wird konnotativ auch dadurch untermauert, dass der Befehlshaber der "légion italienne“ ein Korse ist. Die entsprechenden Geschichten über die der Blutrache verpflichteten und auch ansonsten kaum zivilisierten Korsen von Prosper Mérimée oder Guy de Maupassant aus dem weiteren Verlauf des Jahrhunderts werden dieses französische Klischee bestätigen. Nun ist aber Napoleon ebenfalls Korse, der - und hier wendet der Gesprächsverlauf die Reflexion der Geschichte in eine andere Richtung ganz utilitaristisch die ,ladroni' der italienischen Legion für seine Zwecke einzusetzen weiß und sie sogar zu Helden transformiert. Und so steht dem weiteren vertikalen Verlauf der Diskussion nichts mehr im Wege, wenn der ,grand poète schließlich zum Höhenflug der Mythisierung Napoleons als Lichtgestalt der Zivilisation ansetzt. Aus der schrecklichen Geschichte vom kannibalischen Soldaten der französischen Armee unter korsischer Führung ist in der Debatte in kurzer Zeit das französische Europa in seiner ganzen zivilisatorischen Größe unter Führung des korsischen Kaisers geworden. Viel spannender als die Geschichte des Generals ist für Balzac die Konversation, die diese Geschichte reflektiert und ihr durch die Reflexionen ganz unerwartete Wendungen gibt. Die Kunst des Impliziten der mündlichen Konversation ist die wahre Literatur. Der Gedankenfluss, der dadurch freigesetzt wird, ist der ästhetische, künstlerische Effekt des Impliziten. Der Balzacsche Erzähler führt nicht nur in den beiden Erzählungen ein fortwährendes Gespräch mit dem Leser. Auch in den anderen Romanen der Comédie humaine ist dieses Gespräch durchgängig zu beobachten ${ }^{22}$. Auf diese Weise wird die im 17. Jahrhundert erfolgte Habitualisierung der indirekten Kommunikation des Salongesprächs in der Literatur positiv aufgehoben und weitergeführt.

22 Vgl. dazu Aude Deruelle, „Les Adresses au lecteur chez Balzac“, Cahiers de narratologie Bd. 11/2004, S. 1-12. Vgl. auch Jean Rousset, „L'inscription du lecteur dans La Comédie humaine“, in: J. R., Le Lecteur intime. De Balzac au journal, Paris, Corti, 1986, S. 39-42, Franc Schuerewegen, „Réflexions sur le narrataire”, Poétique H. 70/1987, S. 247-254, Éric Bordas, Balzac, discours et détours. Pour une stylistique de l'énonciation romanesque, Toulouse, Presses universitaires du Mirail, 1997, S. 243-276. 